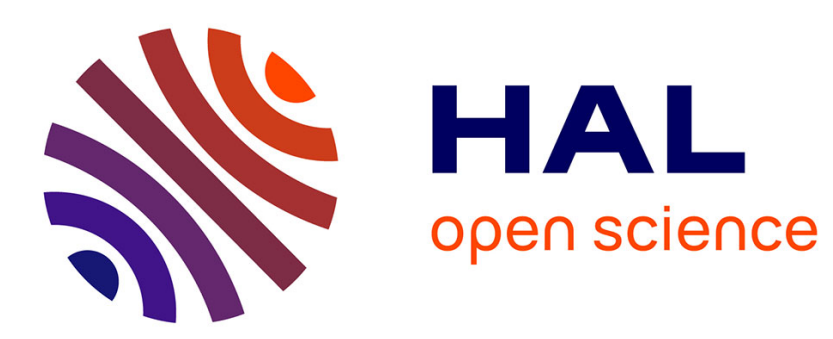

\title{
Dependability evaluation of a GNSS and ECS based localisation unit for railway vehicles
}

Khanh Nguyen, Julie Beugin, Juliette Marais

\section{To cite this version:}

Khanh Nguyen, Julie Beugin, Juliette Marais. Dependability evaluation of a GNSS and ECS based localisation unit for railway vehicles. ITST 2013, 13th International Conference on ITS telecommunications, Nov 2013, Finland. 6p. hal-00930588

\section{HAL Id: hal-00930588 \\ https://hal.science/hal-00930588}

Submitted on 14 Jan 2014

HAL is a multi-disciplinary open access archive for the deposit and dissemination of scientific research documents, whether they are published or not. The documents may come from teaching and research institutions in France or abroad, or from public or private research centers.
L'archive ouverte pluridisciplinaire HAL, est destinée au dépôt et à la diffusion de documents scientifiques de niveau recherche, publiés ou non, émanant des établissements d'enseignement et de recherche français ou étrangers, des laboratoires publics ou privés. 


\title{
Dependability evaluation of a GNSS and ECS based localisation unit for railway vehicles
}

\author{
T.P.K.Nguyen, J. Beugin, J. Marais \\ Univ Lille Nord de France \\ IFSTTAR - COSYS \\ The French Institute of Science and Technology for Transport, Development and Networks, \\ COmponents and SYStems Department, Villeneuve d'Ascq, France \\ Emails: khanh.nguyen, julie.beugin, juliette.marais@ifsttar.fr
}

\begin{abstract}
Today, GNSS-based solutions (Global Navigation Satellite Systems) facilitate the implementation of the train localisation function on-board the vehicle. In the railway context, as a train has to travel different zones on its itinerary, multiple obstacles in these environments can cause different signal perturbations: multipaths, signal delays and masking phenomena that lead to negative consequences on the position accuracy. To reinforce the position quality, a localisation system, developed in the GaLoROI european Project and based on the combination of sensors such as a GNSS receiver and an Eddy Current Sensor, is studied. In this paper, we present a procedure and a model, which aims at evaluating the dependability of this system under local impacts of different railway environments. It allows us analysing complex behaviours of the sensor fusion component on the availability and accuracy of data provided by GNSS \& ECS sub-systems and also to take into account the reliability parameters of hardware components.
\end{abstract}

\section{INTRODUCTION}

One of the keys in automatic train control systems is localisation of railway vehicles. It generally relies on track side components. However, such devices lead to high maintenance costs and also expensive investment costs for infrastructure deployment. For several years, the evolution of the localisation from trackside to trainborne side is a promising solution for this issue. In fact, no infrastructure installation has to be in relation with the on-board system and its maintenance can be easily done during the frequently checks of the trains.

By offering an interoperable worldwide solution, Global Navigation Satellite System (GNSS) becomes an advantageous solution for on-board localisation units. That is the reason why, numerous articles analysed if the performances of GNSS systems satisfy the railway requirements, in particular for safety-related applications. Indeed, using experimental results, [4] concluded that a standalone GPS/GLONASS satellite navigation system and also its combination with inertial navigation systems (INS) do not meet the strong safety-related requirements mentioned in railway standards. This point of view is reinforced in [3]. The authors showed the unavailability probability of the GNSS-based train localisation unit in different zones like tunnels, urban, wooded and railway cutting environments is far to attain the expected safety integrity level and needs to be augmented by other navigation sensors in order to overcome GNSS outage situations. In a recent study, authors of [13] performed numerous test runs on the High Tatra mountain railway line in order to evaluate RAMS parameters of a GNSS based localisation unit. They showed that the performances of GNSS cannot meet the railway requirements in a forest zone and suggested to integrate other on-board localisation sensors.

These above studies emphasized the necessity of reinforcing the performances of GNSS localisation unit by other sensors when they are used in safety applications. However, identifying an appropriate configuration associated to a data combination strategy that meet railway requirements remains an issue. This question is considered in [1]. From the discussion about a short-listing of data fusion options between GNSS signals and other sensors, the authors highlighted one of the advantages of the Eddy Current Sensor (ECS) compared to INS, which is the avoidance of velocity errors due to slip/slide. In fact, the combination between GNSS signals and ECS signals permits to overcome the disadvantages of each component taken separately and to increase the accuracy and robustness of the global localisation unit.

In this context, the GaLoROI project (Galileo Localisation for Railway Operation Innovation), which aims at developing a certifiable, safety-relevant, and satellite-based localisation unit for low density railway lines, is ongoing. The operation principle of GaLoROI is to combine satellite positioning data with satellite-independent data, here provided by an ECS, in order to provide a safe, accurate and continuous train position. The development process of this new localisation system requires to evaluate performances and dependability parameters according to EN50126-1, 2 \& EN 50129 ( [5]-[7]). The dependability parameters, i.e. the Reliability, Availability, Maintainability (RAM) parameters are used in the railway domain to characterize the conditions that maintain the system in a state to deliver a correct required service during operations. In this paper, we present the first step of the dependability assessment process for GNSS \& ECS based localisation unit, the core part of the GALOROI system. The quality of GNSS signals degraded by local phenomena in railway environment and also, the complex behaviours of the sensor fusion, pose multiple challenges for analysing failure causes of the localisation service and for evaluating its dependability. In this article, we propose a new methodology that meets these challenges.

The paper is structured as follow: in Section II, we will describe the system and its components under the dependability aspects. The issues, which concern qualitative and quantitative evaluations of the system dependability, will be presented in 


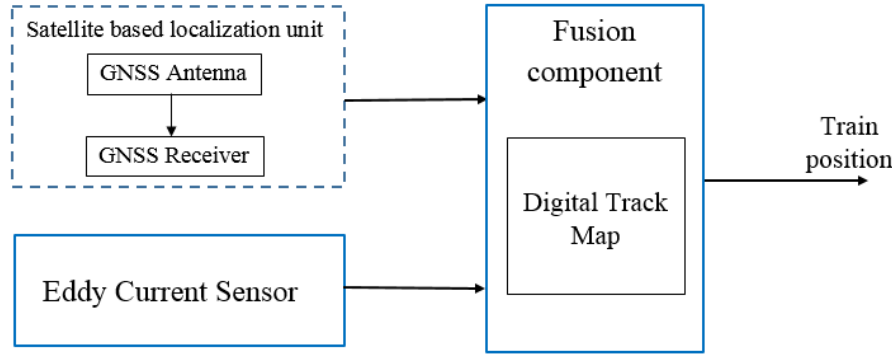

Fig. 1. System concept

Section III. Finally, Section IV presents the conclusion and the future research works.

\section{DESCRIPTION OF THE ON-BOARD LOCALISATION UNIT AND ITS ERROR CONDITIONS}

Figure 1 presents the working principle of the localisation unit. Every data from both GNSS and eddy current sensors contain information about the position and the velocity of the train and are combined in a fusion component. This process is implemented in a computer that integrates a digital track map. With a data fusion algorithm that includes a map-matching process, an accurate train position can be calculated in realtime.

\section{Satellite based localisation unit}

The GNSS antenna is used to collect the data that are emitted from satellites. These data, which contain the satellite ephemeris, a set of parameters that describe the satellite orbit, are sent to the receiver. Based on measurements of the signal propagation time between a satellite and a receiver, a pseudodistance can be calculated. Using the ephemeris and pseudomeasurements, the receiver position is generally obtained in real-time. Normally, 3 visible satellites are required for the position estimation. However, in order to overcome errors due to a clock deviation between a satellite and a receiver, the localisation is performed if there exists at least 4 satellites.

The advantage of GNSS localisation unit is to provide worldwide available and highly accurate measurements that are not prone to drifts in contrast to the INS. However, for the dependability analysis of the satellite navigation, multiple error sources can lead to a poor positioning:

- errors in space such as satellite failure, ephemeris errors, orbit errors,

- errors in propagation environment such as ionospheric $\&$ atmospheric delays, meteor impact, multipath deviations, interference impacts, satellite shadowing,

- failures in user hardware equipment, in particular antenna failures or receiver failures (their corresponding failure rates are hereafter called $\alpha_{a}$ and $\alpha_{r}$ ).

\section{Eddy current sensor}

The second localisation device, the ECS, gives a speed value, a distance value and the direction of the vehicle. In the sensor unit, there are two coil systems that are placed along the rail direction with a fixed distance between them of $L \mathrm{~m}$. Each coil system generates a time varying signal that represents the variation of the eddy currents along the rail. By an analysis of electromagnetic signal correlation, the time delay $(\Delta T)$ between the signals from the two coil system is obtained. The actual speed with which the train runs along the track is then calculated: $v=L / \Delta T$. Furthermore, the direction of the movement can be detected. Thus the ECS measurements are totally independent of the slipping or slidding errors.

The ECS is not affected by weather conditions such as rain, snow, ice, etc, or by pollution. The impact of interferences due to electrical conductors near rails is also non-significant. Therefore,the dependability of ECS device is considered using only its hardware failure rate called $\alpha_{e}$.

\section{Digital track map}

The digital track map is stored in a computer that performs the data fusion function. It comprises all relevant track information such as length of track sections, geo-coordinates to the track elements, hazard areas. These information are customised and updated for particular application area.

The consistency checks for track map information are performed only at the program start of the localisation unit. If any errors are detected, the program is stopped. Therefore, when the program starts successfully, we do not consider the possibility of errors caused by the digital track map.

\section{Fusion component}

The position information coming from the GNSS receiver and from the ECS are sent to the fusion component every $T$ $\mathrm{s}$. Then the fusion component combines these data to ensure reliable positioning results. In [2], an example of data fusion approach for a satellite-based localisation unit is provided and is based on an Extended Kalman Filter. By discussing about the approach limits, the author also suggests an idea that is to use the digital track map as a further source in order to overcome the lack of information in the case of sensor faults. In fact, a data fusion approach combined with a map-matching algorithm is being currently developed in the GaLoROI project [12]. For the dependability analysis in this paper, we do not mention the algorithm and we are only interested in the position results at the fusion component output. By analysing the fusion component behaviours, we find that an estimated position in output of the system is considered as incorrect if one of the following states occurs:

- Unavailable ECS and GNSS data: If there is no ECS and GNSS data for more than $T_{1} \mathrm{~s}$, the output of the fusion can be considered as false.

- Unavailable GNSS data: If GNSS data are missing for more than $T_{2} \mathrm{~s}$ the confidence interval linked to output data will increase quickly. In that case, the position is not trustworthy and considered as false.

- Unavailable ECS data: If the ECS data are missing but GNSS measurements are available, the system can estimate the train position and the process goes on. Note that the inaccurate measurements of the ECS are not considered because no reliable criterion allows us to judge whether a ECS measurement is invalid. 


\section{- Inaccurate GNSS data:}

- At least $k$ consecutive position errors of the receiver that are greater than $x$ meters $\left(P E_{r}>\right.$ $x$ ) can lead to a position error in output of the fusion component that exceeds the user tolerance limit.

- If the ECS data are missing, at least $l$ consecutive position errors of the receiver that are greater than $x$ meters $\left(P E_{r}>x\right)$ can lead to a position error in output of the fusion component that exceeds the tolerance limit.

Note that due to the efficiency of the fusion, the impact of position errors at the receiver output on the global position result will be reduced if there exists valid ECS data, thus $k>l$.

On the other hand, we also consider material failures of the fusion component using the failure rate $\alpha_{f}$.

\section{FIRST STEP OF THE DEPENDABILITY EVALUATION OF THE LOCALISATION UNIT}

The first step of the dependability evaluation is to define, analyse and evaluate the service failures of the system. From railway user's point of view, the positioning function is considered as failed in the following cases:

- Case A - unavailable output of the fusion component. In this case, the localisation service expected by the user is interrupted.

- $\quad$ Case B - untrustworthy position, i.e. the position result has a large estimated confidence interval. In this case, the service is still delivered. However, the position result with its large confidence interval cannot be used in safety-relevant train control applications.

- Case C - estimated position is outside accuracy boundaries. In this case, the localisation service expected by the user is failed but is not recognized by the system or the user.

The combinations of causal events leading to each above mentioned case will be identified by the qualitative analysis presented in the next subsection. The probability of each case will be evaluated in the subsection related to the quantitative analysis.

\section{Qualitative analysis of dependability of the positioning service}

As mentioned previously, the qualitative analysis of the GNSS and ECS-based localisation system encounters multiple challenges because of specific properties of the GNSS signals and because of complex behaviours of the fusion component.

On the one hand, common analyses of dependability cannot adaquately take all perturbations affecting GNSS signals into account, especially local impacts of railway environments. In order to overcome this difficulty, we model the GNSS receiver output using 4 states:

1) Correctly estimated position, i.e. when the difference between the true position, unknown for the user, and the estimated position is inferior than a tolerance limit laid down by user requirements: $P E_{r} \leq x \mathrm{~m}$.

2) Incorrectly estimated position, i.e. when the estimated position is outside accuracy boundaries: $P E_{r}>x$ $\mathrm{m}$. Consecutive position errors can lead to a failed service of the global localisation unit.

3) Position is not delivered because, at the receiver input, the number of valid signals received are insufficient (Missing-GNSS-signal).

4) Position is not delivered because of an hardware failure.

In the next subsection, the transition probabilities between these states can be identified using data collected from:

- simulations [3],

- real tests [13].

Note that in both articles [3], [13], the authors only focus on the important impact of local phenomena and do not consider the hardware failures. This aspect is also examined in our paper with relevant hardware failure rates.

On the other hand, a new approach that allows the analysis of the dynamic behaviours of the fusion component is required. This approach has to consider at each sampling instant if sensor data are available and accurate, and has also to handle temporal dependencies.

In reliability and safety studies, the traditional fault tree method (FT) [8] is widely used because it is suitable for both qualitative and quantitative analyses. In fact, it provides an ideal framework for deductive analyses that look for various possible combinations of causal events leading to the top event (feared event). It also allows the calculation of probabilities related to the combinatorial logic gates. However, this method is not sufficient to capture behaviours and interactions of components of complex and hybrid systems that integrate both continuous and discrete dynamic behaviours. The Dynamic Fault Tree method (DFT) [14] is an extension of the FT method by defining additional dynamic gates in order to attain a higher level of systems' dependability analysis. This method allows the analysis of failure sequences, functional dependent failures or priorities on failure events. Moreover, the FT method with time dependencies between events (TdFT) [10] is also useful. It allows duration conditions leading to hazards to be considered inside logic gates. In order to combine advantages of the above methods aiming at capturing the fusion component behaviours, we present in this subsection a hybrid fault tree model.

Let us consider the FT in Figure 2, which analyses failed outputs of the fusion component, those failures intervene in the three cases A, B and C. The unavailable output (Case A) is caused by a material failure (Basic Event 1 - BE1) or by a software error in the fusion component (Undeveloped Event UE). The material failure occurs with a failure rate $\alpha_{f}$ while the software error is not analysed in the framework of this paper.

The untrustworthy position (Case B) can be caused by a lack of both GNSS and ECS data for more than $T_{1} s$ (called Intermediate Event 1 - IE1) or by missing GNSS data for more than $T_{2} s$ (Intermediate Event 2 - IE2). 


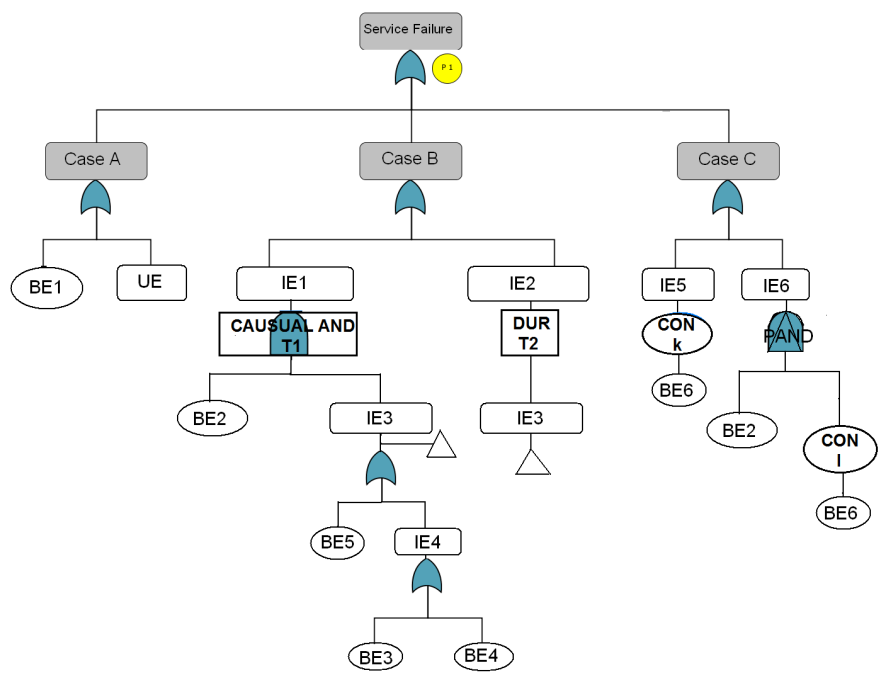

Fig. 2. Hybrid Fault Tree of service failure

BE1: material failure of the fusion component

BE2: ECS failure

BE3: antenna failure

BE4: receiver failure

BE5: missing GNSS signal (signal in space)

BE6: position error at the receiver output $>x \mathrm{~m}$

IE1: lack of both GNSS and ECS data for more than $T_{1} \mathrm{~s}$

IE2: missing GNSS data for more than $T_{2} \mathrm{~s}$

IE3: missing GNSS data

IE4: GNSS hardware failure

IE5: at least $k$ consecutive position errors of the receiver $>x \mathrm{~m}$

IE6: at least $l$ consecutive position errors of the receiver $>x \mathrm{~m} \mid$

ECS fails

UE: software error in the fusion component

Next, the IE1 is the output of a causal AND gate (defined in [10]) having in input ECS failure (Basic Event 2 - BE2) and missing GNSS data (Intermediate event - IE3) with a duration greater than $T_{1} \mathrm{~s}$. The output of the causal AND gate only happens when its inputs occur together during the given period of time. The IE3 has in input the BE5 - Missing of GNSS signal (state 3 of receiver output) or IE4 - GNSS hardware failure (state 4 of receiver output) caused by an antenna failure (BE3) or receiver failure (BE4). Similarly, the IE2 is a duration gate output (DUR gate) having in input the IE3 for more than $T_{2}$ s. The DUR gate is defined by the occurrence duration of the input during a given period of time.

The case $\mathrm{C}$ is caused by IE5 - at least $k$ consecutive $P E_{r}>$ $x m$ or IE6 - at least $l$ consecutive $P E_{r}>x m$ when ECS fails. We define in this paper the consecutive gate (CON gate). Its output only happens when its input consecutively occurs at least $N$ times. The IE5 is the CON gate output of $k$ consecutive events $P E_{r}>x m$ (BE6, state 2 of the receiver output). Next, the IE6 is characterized by the priority-AND gate (PAND gate defined in [14]) of a ECS failure (BE2) and a CON gate of $l$ consecutive events $P E_{r}>x m$ (BE6). The PAND gate output only happens when its inputs occur from left to right.

With a dynamic gate (PAND gate) and time dependency gates (Causal AND gate, DUR gate, CON gate), the proposed hybrid fault tree allows the complex behaviours of the fusion component to be captured and characterized. Then, the causes

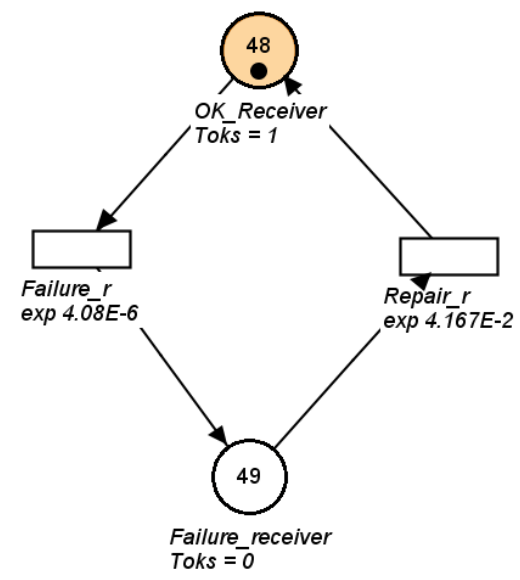

Fig. 3. DSPN structure for hardware states of components

that lead to failed outputs can be studied. However, the analytical evaluation of the system availability in terms of a structure function is a complex issue and is not performed within the framework of this article. For the quantitative analysis, the service failure probability will be evaluated by another approach presented in the next subsection.

Quantitative analysis of dependability of the positioning service

Based on the hybrid fault tree presented before, the Dynamic Stochastic Petri Net (DSPN) is used to quantitatively analyse service failures. This method is widely employed in dependability assessments and allows time dependent behaviours in a system to be taken into account. An event transition in a DSPN can occur according to the three following ways:

- immediately when all guard conditions are satisfied (type 1)

- $\quad$ after a constant delay (type 2)

- after an exponentially distributed probabilistic delay (type 3)

The quantitative analysis is performed according to the three following steps:

- Step 1 - model the evolution of sub-system states over time.

The hardware states of ECS, antenna, receiver, fusion component is modelled by two PN places: $O K$ and Failure places (Figure 3 illustrates the receiver example). Their failure events and reparation actions are characterized by two transitions of type 3 associated to the corresponding failure rates (see values in Table I) and with a reparation rate of $1 / 24 \mathrm{~h}$.

Based on the qualitative analysis, the 4 states of the receiver output are modelled in the DSPN illustrated in Figure 4. The transitions between the states 1/2/3 only occur when no material failure exists. Their probabilities are calculated from the simulation data used in [3]. The transitions from these three states to state 4 (hardware failure state) immediately occur 


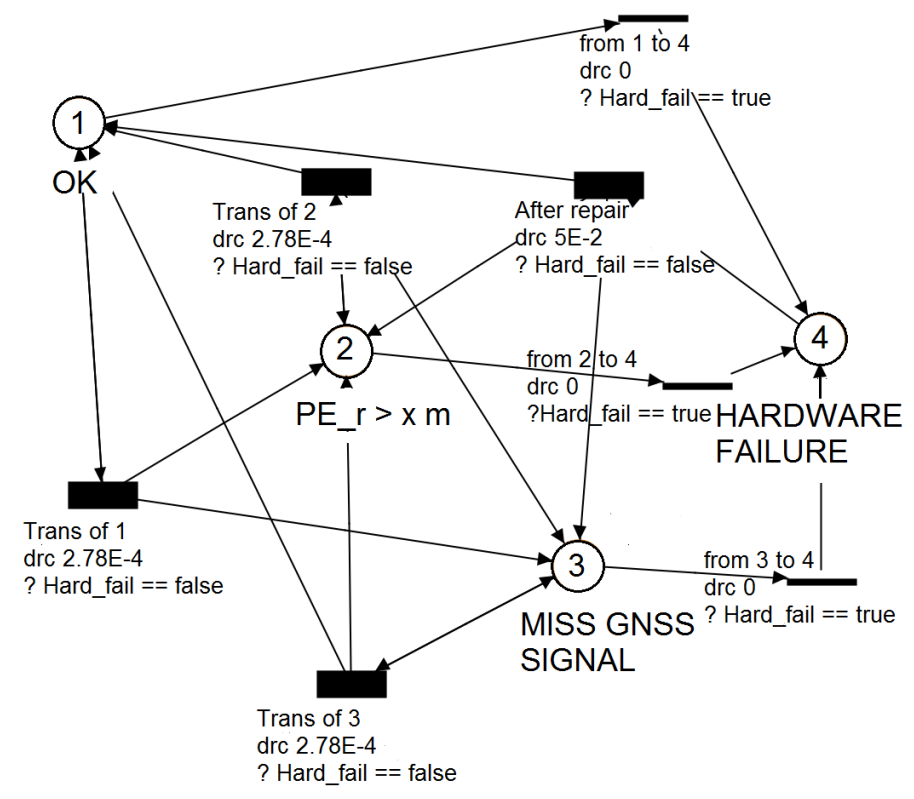

Fig. 4. DSPN structure for position results of GNSS receiver

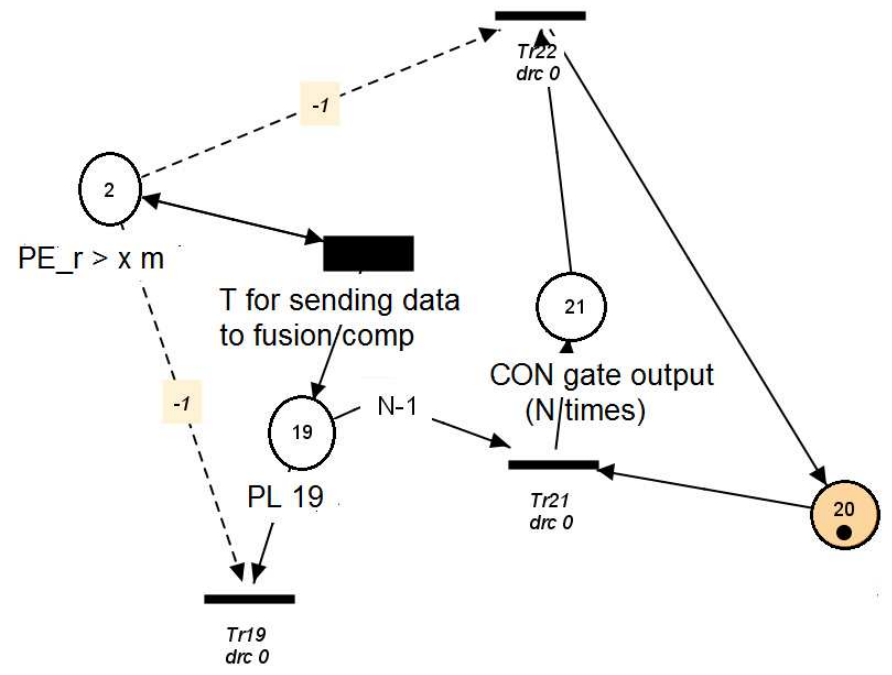

Fig. 5. DSPN structure for CON gate of $\mathrm{N}$ consecutive events

when there exists at least a material failure of a component. Finally, after a reparation action, if all components are $\mathrm{OK}$, the transition from state 4 to one of the three states $1 / 2 / 3$ is fired after the time to first fix (TTFF) of the receiver.

- $\quad$ Step 2 - model dynamic logic gates.

The translation of OR, PAND, DUR gates to the corresponding DSPN structure is proposed in [11]. The Causal AND gate is modelled by a combination of AND and DUR gate. Finally, we propose in this paper the CON gate model. It is described in Figure 5.

- $\quad$ Step 3 - model and evaluate the global behaviour of the system.
The probability of the top event of the hybrid fault tree is evaluated using the DSPN simulation results.

TABLE I. INPUT PARAMETERS FOR EVALUATING THE FAILURE SERVICE OF SYSTEM

\begin{tabular}{ccccc}
\hline Missing time \& Number & $T_{1}$ & $T_{2}$ & $k$ & $l$ \\
\cline { 2 - 5 } of consecutive PE & $3 \mathrm{~s}$ & $60 \mathrm{~s}$ & 2 & 6 \\
\hline Failure rate $\left(/ 10^{-6} \mathrm{~h}\right)$ & $\alpha_{a}$ & $\alpha_{r}$ & $\alpha_{e}$ & $\alpha_{f}$ \\
\cline { 2 - 5 } & 4 & 4.08 & 2 & 6.06 \\
\hline
\end{tabular}

TABLE II. PROBABILITY OF SERVICE FAILURE IN DIFFERENT ENVIRONMENTS

\begin{tabular}{|c|c|c|c|c|}
\hline & Urban & Tunnel & Woody & $\begin{array}{l}\text { Railway } \\
\text { Cutting }\end{array}$ \\
\hline Service failure & $1.65 E^{-4}$ & $1.53 E^{-2}$ & $7.59 E^{-2}$ & $7.17 E^{-4}$ \\
\hline $\begin{array}{l}\text { Fusion } \\
\text { component } \\
\text { failure }\end{array}$ & \multicolumn{4}{|c|}{$3.42 E^{-6}$} \\
\hline $\begin{array}{l}\text { Missing GNSS } \\
\text { during 60s }\end{array}$ & $4.1 E^{-6}$ & $1.53 E^{-2}$ & $4.1 E^{-6}$ & $4.1 E^{-6}$ \\
\hline $\begin{array}{l}\text { Missing ECS \& } \\
\text { GNSS during } 3 s\end{array}$ & $8.34 E^{-11}$ & $6.63 E^{-7}$ & $7.4 E^{-10}$ & $3 E^{-9}$ \\
\hline $\begin{array}{l}\text { Failed ECS \& } \\
2 \text { consecutive } \\
P E_{r}>50 \mathrm{~m}\end{array}$ & $1.63 E^{-8}$ & 0 & $2.73 E^{-7}$ & $2.95 E^{-8}$ \\
\hline $\begin{array}{l}6 \quad \text { consecutive } \\
P E_{r}>50 \mathrm{~m}\end{array}$ & $1.57 E^{-4}$ & 0 & $7.59 E^{-2}$ & $7.1 E^{-4}$ \\
\hline
\end{tabular}

TABLE III. COMPARAISON OF THE AVERAGE AVAIBILITY OF ECS AND GNSS LOCALISATION UNIT AND SINGLE GNSS LOCALISATION UNIT [3]

\begin{tabular}{c|c|c}
\hline Environment & ECS \& GNSS unit & Single GNSS unit \\
\hline Railway Cutting & $99.93 \%$ & $87.76 \%$ \\
\hline Urban & $99.98 \%$ & $93.88 \%$ \\
\hline Woody & $92.41 \%$ & $60.23 \%$ \\
\hline Tunnel & $98.45 \%$ & $15.57 \%$ \\
\hline
\end{tabular}

There are numerous tools to create and evaluate a DSPN. In this paper, we use the Petri Net module of GRIF platform [9] to illustrate the performance of our model. The dependability assessment of the global localisation unit on a mission of 1 hour is based on the following assumptions:

- The system is considered to be fault free at the start of the mission.

- Failed components are repaired after the tests $(\mu=$ $1 / 24 \mathrm{~h}$ ) and the TTFF of the global system is $180 \mathrm{~s}$.

- Consecutive position errors of GNSS receiver output of more than $50 \mathrm{~m}$ can lead to a position error of the global system (estimated position is outside accuracy boundaries).

- Other parameters that characterize system behaviours are given in Table I.

Table II gives the results of the quantitative analysis for the GNSS and ECS based localisation system. The probabilities of intermediate events (IE) that directly lead to the Top event (TE) are respectively presented in the right part of the Table. By considering the probability of TE (service failure) and IEs, we see that the principal cause of a service failure is the 
impact of the operational environment on the GNSS output. For example, in woody environment, the IE5 (more-than6-consecutive-GNSS-PEs) with an occurrence probability of $7.59 E^{-2}$ is the critical event that leads to the global service failure. In tunnel environment, the occurrence probability of IE5 is reduced and is non significant while the IE2 (missingGNSS-data-for-more-than-60s) with its occurrence probability of $1.53 E^{-2}$ becomes the principal cause of TE.

By considering Table III, the service availability of global system are improved significantly when compared with a single GNSS-based localisation unit at the accuracy level of 50 $\mathrm{m}$ [3], especially in areas of low GNSS signals. For example, in tunnel environment, the service availability is reinforced from $15.57 \%$ to $98.47 \%$.

\section{CONCLUSION}

On-board localisation equipment in railway systems can beneficially evolve using GNSS. Moreover, the combination of GNSS sensors to ECS sensors can improve significantly the positioning quality in case of GNSS outage situations. However, such configuration poses numerous challenges when analysing and evaluating the system dependability. We have presented in this paper a hybrid fault tree model aiming at performing the dependability assessment of a GNSS-based and ECS-based localisation unit. Using dynamic gates (PAND gates) and new time dependency gates (Causal AND gates, DUR gates and CON gates), the hybrid fault tree method is powerful for analysing complex behaviours of numerous systems.

For the qualitative evaluation of dependability of the studied system, the method analysed dynamic and time-dependent behaviours of the data fusion. Additionaly, the model of the receiver outputs considers local impacts of different railway environments and the hardware failure probability.

The quantitative analysis was implemented by translating the elements of the hybrid fault tree toward DSPN. Then, we used an existing tool -the Petri net module of GRIF platform in order to evaluate the probability of the global service failure. The results illustrated the efficiency of integrating an ECS into a satellite-based localisation unit. However, for a safety application ensuring a safe railway traffic, this configuration is not sufficient as the safety requirement for the localisation function is not met. It has to be reinforced by a redundant equipment. This principle is adopted for the GaLoROI system.

These above results are preliminary conclusions obtained using the illustrated numerical example of [3] based on simulation data. As the system is totally new and is still developing, real data are not yet available to tune the model of the system. In future work, after the system tests in operational environments will be completed, we will analyse experimental data and will apply them into the model for RAMS assessments. Furthermore, a more efficient algorithm to improve the implementation time of Petri Net for quantitative evaluation could also be developed.

\section{ACKNOWLEDGEMENTS}

This research was conducted as part of the GaLoROI project (Galileo Localisation for Railway Operation Innovation) supported by the European commission. GaLoROI is an integrated research project within the European 7th Framework Programme.

\section{REFERENCES}

[1] A. Acharya, S. Sadhu \& T.K. Ghoshal, Train localization and parting detection using data fusion, Transportation Research Part C 19, 2011, 75-84

[2] F. Boehringer, Train location based on fusion satellite and train-borne sensor data, Proc. SPIE 5084, Location Services and Navigation Technologies, 76 (August 6, 2003); doi:10.1117/12.487062

[3] J. Beugin, J. Marais, Simulation-based evaluation of dependability and safety properties of satellite technologies for railway localization, Transportation Research Part C 22, 2012, 42-57.

[4] A. Filip, L. Bazant, H. Mocek \& J. Cach, GPS/GNSS based train position locator for railway signalling, Computers in Railways VII, 2000, ISBN $1-85312-826-0$

[5] EN 50126-1, 2000. Railway applications specification and demonstration of reliability, availability, maintainability and safety (RAMS) Part 1. CENELEC European standard (European Committee for Electrotechnical Standardization).

[6] EN 50126-2, 2007. Railway applications specification and demonstration of reliability, availability, maintainability and safety (RAMS) Part 2: Guide to the application of EN50126-1. CENELEC European technical report (European Committee for Electrotechnical Standardization).

[7] EN 50129, 2003. Railway applications communication, signalling and processing systems safety related electronic systems for signalling. CENELEC European standard (European Committee for Electrotechnical Standardization).

[8] Fault Tree Handbook, U.S. Nuclear Regulatory Commission, Washington, DC, 1981, NUREG-0492.

[9] GRIF - GRaphical Interface for reliability Forecasting, http://grifworkshop.com/grif/petri-module/

[10] J. Magott, P. Skrobanek, A Method of Analysis of Fault Trees with Time Dependencies, Computer Safety, Reliability and Security Lecture Notes in Computer Science Volume 1943, 2000, pp 176-186.

[11] B. Kaiser, C. Gramlich, State-Event Fault Trees - A Safety Analysis Model for Software Controlled Systems, Computer Safety, Reliability, and Security Lecture Notes in Computer Science Volume 3219, 2004, pp 195-209.

[12] M. Lauer, D. Stein, Algorithms and Concepts for an Onboard Train Localization System for Safety-Relevant Services, IEEE ICIRT - International Conference on Intelligent Rail Transportation, Beijing, China, 2013, 6p.

[13] D.Lu, F. G. Toro and E. Schnieder, RAMS Evaluation of GNSS for Railway Localisation, ICIRT 2013 - IEEE International Conference on Intelligent Rail Transportation, Beijing, China, August 2013.

[14] G. Merle, Algebraic modelling of Dynamic Fault Trees, contribution to qualitative and quantitative analysis, $\mathrm{PhD}$ thesis of Ecole Normal Supérieur de Cachan, 2010. 